\title{
INTRODUCTION
}

\section{The immediate global impact of COVID-19 on higher education institutions and workforce development}

\author{
Mrs Lawrencia Louise Brown \\ Research Department of Practice and Policy, UCL School of Pharmacy, London, United Kingdom
}

\begin{abstract}
As the World Health Organisation (WHO) made us aware of the emergence of the COVID-19 virus and its progress across the regions of the world, it triggered the creation of initial plans for a potential response by governments and healthcare related organisations.
\end{abstract}

In January 2020 The World Health Organisation (WHO) mission to Wuhan, China issued a statement suggesting the possibility of human to human transmission of the COVID-19 virus. This became a major factor in advancing these plans and started the focus on situations where large numbers of people come into close contact with each other and how those transmission rates could be reduced. Within higher education institutions (HEls) this quickly evolved, in many cases due to direct governmental intervention, into the suspension of all face-to-face activities within HEls - including teaching, assessment and research.

Pharmacy academics had to rapidly respond to these changing conditions - assessing how and what, should or could be taught by remote methods. Consideration of what remote methods institutions had previously funded or were willing to fund varied by institution, but it appears that universities which, pre-COVID-19, had been in the fiscal position to invest in such technologies and IT teams to support them were able to give their academic staff more options to adapt their teaching and assessment offering. The cases in this report describe the use and adaption of a wide range of specialist learning technologies in an attempt to deliver on the same or modified learning outcomes for their students in engaging and innovative ways.

Many institutions opted to deliver synchronous teaching sessions through the use of video conferencing software such as Zoom or Microsoft Teams, with large timetabled sessions that often monitored: attendance, student's behaviour when in smaller groups created within virtual breakout spaces, and use of the chat function in order to determine a general sense of student engagement in these sessions.
Some HEls showed their versatility by using more widely available social media apps such as WhatsApp as a platform for asynchronous discussion or provision of feedback on assignments between students, academic staff and in some cases, as a means by which preceptors or pharmacist tutors could also connect. The advantage of using such platforms is that the majority of students were likely to already have them and understand how they can be used.

But the rapid switching from traditional methods of teaching to modes of delivery that require access to high speed broadband, access to a smart phone, tablet device or laptop was not without issues. Some institutions reported that when they released their new plans for teaching and assessment during COVID-19, student feedback highlighted that access to these resources was by no means universal and in certain circumstances a significant barrier that required a novel and sometimes low-tech approach by pharmacy academics. Examples such as increasing the time window in which online assessments could be completed, allowing multiple attempts in completing online quizzes so any issues with internet connectivity could be accommodated, and ensuring students had access to any support materials required in various ways were employed.

In many of the cases included in this collection you will find examples of pharmacy academics involving students in various aspects of their immediate response to COVID-19. The use of student evaluations to gain feedback in these newly designed teaching sessions and to new forms of assessment was often employed, and provided an important source of reflection for pharmacy academic teams as they navigated this continually evolving situation.

In some instances, feedback highlighted that students felt that not all online activities, however well planned and executed, could replace traditional modes of learning such as wet laboratory sessions or experiential placements in healthcare environments. There was mention not only of 
difficulty in the development of hands-on skills and behaviours, but also that these activities were often designed as a place where students could consolidate and apply knowledge that had been delivered earlier in more traditional modes such as lectures. In addition, reference was made to students missing the socialisation that occurs within the university environment.

Across many platforms, including social media, the experiences were shared of academics within all areas of healthcare, including pharmacy, who as registered practitioners decided to come back in some capacity into patient facing roles. In some instances this movement of workforce grew as more people returned to patient facing practice as the impact of COVID-19 on the populations within their country grew in size and aggressiveness. Taking a deeper look at what the impact was on those pharmacists would be an important area of review. It would be of interest, and useful, to find out how their HEls responded to releasing them back into practice, to what extent they worked, and what the psychological effect of delivering frontline pharmaceutical care during a pandemic have been. It is important to identify and understand how, or if, this has, or will, impact on what they teach pharmacy students. Would they want to explore more fully the discipline of global health, responses to large scale medical crises, the role of $\mathrm{WHO}$ and the health inequalities that exist in all countries and communities?

Some HEl's pharmacy departments have been measuring the impact on academic staff working through COVID-19, looking at how they have reacted to the abrupt moving to remote working, the increase in working online (especially in delivering teaching and assessments) and in adjusting to using current learning technologies in a new way - such as the use of the quiz function on Goggle Forms or learning technologies that were brand new to them such as Zoom or Mentimeter. This may be important going forward as many countries potentially face a further wave of COVID-19 infections and more governmental restrictions that will undoubtably follow.

Pharmacy academics have had to show flexibility of approach, a great deal of personal resilience, a strong focus on team working, and a willingness to engage with 'out of the box thinking' in order to drive innovation. Re-defining what is taught and assessed for their students in this changing landscape, within the confines of what delivery methods are open to them, all while having to align with their government and HEl's restrictions on social distancing.

The additional workload needed, especially in the early weeks and months of lockdown, naturally varied according to where in the academic year pharmacy departments were when COVID-19 reached their country, but it would be acceptable to say that it has been a time of significant impact for pharmacy academics and the wider staff involved in delivering courses within HEls.
The cases in this collection represent some of the innovative work the global community of pharmacy academics have been engaging in, and that they wish to share with you - in the hope that it will inform the potential curricula and course re-design that HEls will be engaging in over the coming academic year.

We hope the case studies will spark a process of reflection for pharmacy HEls, both for teams and individual pharmacy academics to understand what they can learn from our profession's global response to COVID-19 and how it might shape their future plans.

This collection may prompt HEls to consider what learning technologies may be required as a minimum for future course delivery - e.g. the establishment of an all purpose learning platform such as Moodle or Blackboard, if they do not already have one.

Our collection of case studies should stimulate a discussion about what we teach pharmacy students during their time with us. We now have an opportunity to reflect on how well the courses we deliver truly prepare students to deliver pharmaceutical care in the workplace and whether it allows the development of the broader personal development skills which pharmacists draw upon when working in such challenging times.

Going forward, if HEls are intending to continue the widespread use of online teaching methods and online assessment, then consideration should be given to the building of the IT skills of both staff and students through targeted training and the support of experts where possible. This will also require considering the financial implications of ensuring that everyone has access to the IT infrastructure they need so they are not excluded from learning due to their socio-economic status.

Mrs Lawrencia Louise Brown

Research Department of Practice and Policy UCL School of Pharmacy

Room 338

29-39 Brunswick Square

London WC1N $1 A X$

United Kingdom

lawrencia.brown@ucl.ac.uk 